\title{
Longitudinal monitoring of the dynamics of infections due to Bartonella species in UK woodland rodents
}

\author{
R. J. BIRTLES ${ }^{12 *}$, S. M. HAZEL ${ }^{34}$, M. BENNETT ${ }^{4}$, K. BOWN ${ }^{34}$, \\ D. RAOULT ${ }^{2}$ AND M. BEGON ${ }^{34}$ \\ ${ }^{1}$ Department of Pathology and Microbiology, School of Medical Sciences, University of Bristol, \\ Bristol BS8 1TD, UK \\ ${ }^{2}$ Unité des Rickettsies, UPRESA 6020, Faculté de Médecine, 27 Boulevard Jean Moulin, \\ 13385 Marseille cédex 5, France \\ ${ }^{3}$ Population Biology Research Group, School of Biological Sciences and Centre for Comparative Infectious \\ Diseases, University of Liverpool, PO Box 147, Liverpool L69 3BX, UK \\ ${ }^{4}$ Department of Veterinary Clinical Science and Animal Husbandry and Centre for Comparative Infectious \\ Diseases, University of Liverpool, Leahurst, Chester High Road, Cheshire CH64 7TE, UK
}

(Accepted 14 November 2000)

\section{SUMMARY}

Blood samples were repeatedly collected from 12 sympatric woodland rodents over a 12-month period and DNA extracts from each were incorporated into a bartonella-specific PCR targeting a fragment of the $16 \mathrm{~S} / 23 \mathrm{~S}$ rRNA intergenic spacer region (ISR). The composition of each amplicon was analysed using restriction enzyme analysis (REA) and base sequence comparison. Bartonella DNA was detected in 70 of 109 samples. Eleven samples contained DNA derived from more than one strain. Sequence analysis of 62 samples found 12 sequence variants (ISR genotypes) that were provisionally assigned to 5 different species, 2 of which were newly recognized. Up to five different species were detected in each animal. On about two-thirds of occasions, a species detected 1 month was not there the next, but never was a genotype superseded by another of the same species. However, a genotype could be re-encountered months later in the same animal, even if interim samples contained other genotypes. Our results suggest that although most animals are bacteraemic most of the time, specific infections are often superseded and that a complex and dynamic epidemiology of bartonella bacteraemias exists in woodland rodents.

\section{INTRODUCTION}

The potential role of parasites and pathogens in the population dynamics of their hosts is now becoming more widely recognized [1]. However, although there is little doubt that most natural populations support endemic microparasite infections that have no obvious or widespread effects on mortality, empirical data to clarify the importance of such infections for the dynamics of their hosts have only recently begun to

* Author for correspondence: Department of Pathology and Microbiology, School of Medical Sciences, University of Bristol, Bristol BS8 1TD, UK. emerge. Bartonella species, which have been demonstrated to parasitize a high proportion of the woodland rodent population in the UK and elsewhere, potentially provide very useful model for addressing this shortfall.

Bartonella species are Gram-negative cocco-bacillary Proteobacteria that parasitize a range of mammalian hosts including man. To date, 13 species have been formally proposed and for most a reservoir, in which infection appears not to induce recognizable disease, has been identified [2-5]. Infections due to Bartonella species can be transmitted between hosts by ectoparasites, and a range of arthropods has been 
implicated including fleas, lice, sandflies and ticks $[2,6]$. Surveys of small woodland mammals in the UK and elsewhere have demonstrated a high prevalence of bartonella within circulating erythrocytes, with, typically, $40-60 \%$ of animals yielding isolates on blood culture [7, 8]. In the UK, four Bartonella species have so far been identified among isolates recovered from bacteraemic animals; these are not host-specific among sympatric small mammal species [7,9]. Furthermore, almost all animal populations studied to date concurrently support multiple species of Bartonella [7, 8].

Previous surveys to detect and characterize Bartonella species in small mammals, however, have been horizontal. The only longitudinal study in UK woodland rodents to date used non-specific staining to identify intra-erythrocytic infections [10] and, while able to demonstrate that infections were encountered throughout the year and that a seasonal variation in prevalence occurred, this study did not attempt to differentiate between the Bartonella species observed. Hence, there are many fundamental but unanswered questions. Is the high prevalence of infection an indication that infection is chronic or is there a rapid turnover of acute infections? Do Bartonella species commonly coexist within an individual host as well as within host populations? Does infection with one Bartonella species, or strain within a species, afford protection against subsequent infection with other species or strains?

Longitudinal sampling of small rodents for bartonella-induced bacteraemia is feasible only if small volumes of blood can be collected without detriment to the animal. The fastidious nature of Bartonella species requires that if culture-based surveillance schemes are to be employed, blood samples be aseptically collected, but in practical terms, such a manipulation in the field is difficult. The advent of PCR-based DNA amplification methodologies has circumvented the requirement to isolate an organism as a means for its detection and as a prerequisite to its identification. Several PCR-based approaches to the detection of bartonellae in clinical and veterinary samples have been described [2]. We have recently evaluated the use of one of these methods in conjunction with subsequent analysis of amplification products for the detection and differentiation of strains of bartonellae causing bacteraemia in woodland rodents [11]. In the current study we have applied this methodology to a preliminary longitudinal survey of wild rodents in two British woodland populations.
The results suggest a complex dynamic of infection and successive re-infection with a diverse range of Bartonella species and strains.

\section{MATERIALS AND METHODS}

The samples used in this study were collected from sympatric Clethrionomys glareolus (bank vole) and Apodemus sylvaticus (wood mouse) populations inhabiting two mixed woodland sites on the Wirral in North West England: Manor Wood and Rake Hey. Sampling of these communities began in March 1995, using methods described in detail elsewhere [12]. Briefly, animals were caught in traps placed at permanent trapping stations 10 metres apart arrayed as a $100 \mathrm{~m}^{2}$ square grid. Each month, two traps were set at each trapping station over three consecutive nights. Traps were checked each morning during the monthly trapping period and all captured animals were scanned for the presence of an electronic transponder (Avid). Newly encountered animals were identified to species, weighed, sexed and tagged. The tail-tip of each animal was then clipped and a few drops of blood were collected into a sterile Eppendorf tube prior to the animal's release. Tagged animals that had not previously been encountered during the monthly trapping period were also weighed and sexed, and a blood sample collected. Finally, tagged animals, which had already been encountered during the ongoing trapping period were immediately released.

All blood samples were centrifuged and serum was removed. The remaining cell pellets were stored at $-20{ }^{\circ} \mathrm{C}$. Testing of all blood pellets collected in August and September 1996 for the presence of bartonella DNA allowed infected animals in those samples to be identified, and 12 of these were selected on the basis of the best availability of samples from the subsequent 12 months. In total, 109 blood samples were tested from these animals, with between 7 and 12 samples being available for each for the period between August 1996 and July 1997.

DNA extracts were prepared from the blood pellets [13] and subjected to a bartonella genus-specific PCR, based on the oligonucleotide primer pair QHVE1 and QHVE3 [14]. The success of each reaction was determined by examination of UVirradiated $1 \%$ agarose gels containing ethidium bromide on which amplification products had been electrophoretically resolved. Products were then assessed by restriction endonuclease analysis (REA); $25 \mu \mathrm{l}$ of product was mixed with $4 \mu \mathrm{l}$ of $10 \times$ reaction 


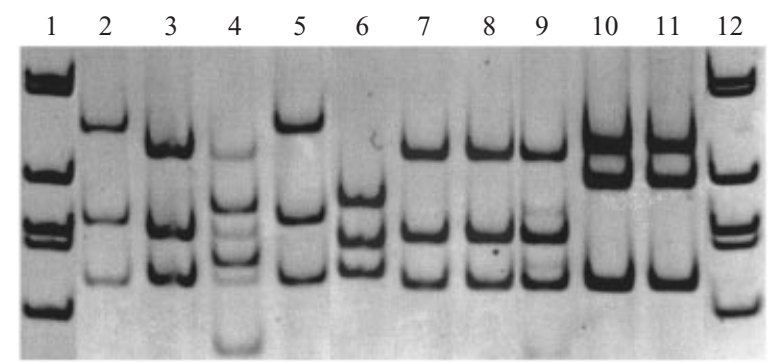

Fig. 1. Restriction profiles obtained following resolution of HaeIII digests of QHVE1/3 amplification products obtained from animal 12. The profiles obtained are as follows: lane 2, N40 (genotype A6); lane 3, B. taylorii (A1 or A5); lane 4, B. taylorii (A1 or A5) and Bartonella sp. (B); lane 5, N40 (genotype A6); lane 6, B. doshiae (C2); lane 7, B. taylorii (A1 or A5); lane 8, B. taylorii (A1 or A5); lane 9, B. taylorii (A1 or A5) and (faintly) Bartonella sp. (B); lane 10, B. grahamii (B); lane 11, B. grahamii (B). Lanes 1 and 12 contain molecular weight markers, with bands at 154 base pairs (bp), $220 \mathrm{bp}, 234 \mathrm{bp}, 298 \mathrm{bp}, 394 \mathrm{bp}$ and $453 \mathrm{bp}$ (molecular weight marker VI, Boehringer Mannheim).

buffer, $1 \mu \mathrm{l}(10 \mathrm{U})$ of HaeIII and $10 \mu \mathrm{l}$ of distilled water then incubated at $37^{\circ} \mathrm{C}$ for $3 \mathrm{~h}$. Digestion products were electrophoretically resolved on an $8 \%$ polyacylamide gel (mini Protan II, BioRad) at $100 \mathrm{~V}$ for $1 \mathrm{~h}$ then visualized by UV illumination following ethidium bromide staining.

The remainder of each amplification product was purified (QIAquick kit, Qiagen) and its base sequence was determined using cycle sequencing reactions containing either QHVE1 or QHVE3 as previously described [11]. Alignment of the partial ISR sequences obtained above with one another and with ISR sequences of the characterized Bartonella species was attempted using version $\mathrm{V}$ of the CLUSTAL multisequence alignment program [15]. The hypervariablity of ISR sequences belonging to different Bartonella species prevented their accurate alignment and thus quantitative comparison of these data could not be made. However, if a new ISR sequence was encountered, an attempt to infer the phylogenetic position of the bartonella strain from which it was derived was made by citrate synthase gene ( lt $A$ ) analysis. Briefly, an approximately 700 base pair 3' fragment of the gene was amplified using PCRs incorporating the previously described primers 440f/1137r [9]. The base sequences of the products obtained were determined then compared ( $\%$ similarities calculated) with partial gltA fragments available for previously characterized Bartonella species and strains.

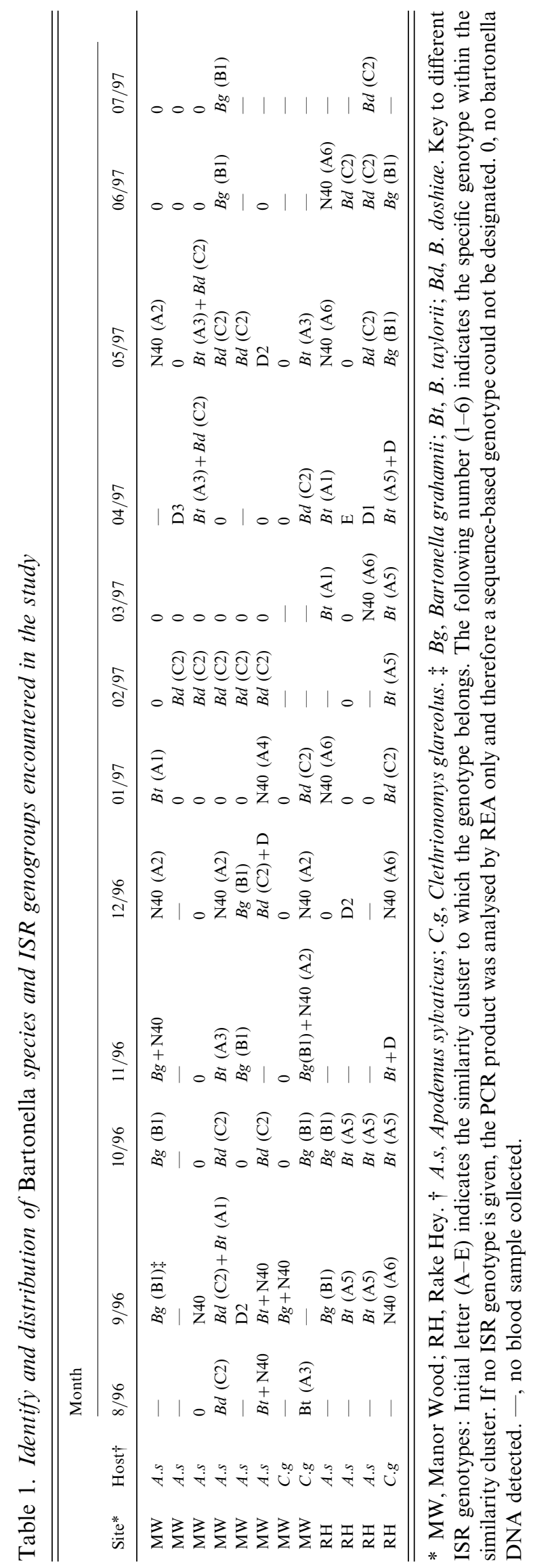




\section{RESULTS}

Preliminary work demonstrated that HaeIII-based REA of ISR amplification products was clearly able to differentiate between the type strains of the four species of Bartonella currently associated with UK woodland rodents and thus that this methodology provided a useful means for assessing the presence of mixed species infections in individual blood samples.

A total of 70 of 109 blood pellets yielded amplification products. REA demonstrated 59 to comprise a single profile and 11 to comprise two superimposed profiles. Most of these 11 mixed profiles consisted of strong bands derived from one profile and weaker bands from a second (Fig. 1). Three previously unrecognized profiles were observed. Sequence analysis was attempted on all amplification products. Nonsensical data were obtained from 8 samples, all of which had yielded mixed profiles on REA but data for the remaining 62 samples were unambiguous (Table 1).

A total of 12 different sequences were obtained. Five of these had been encountered previously among sequences derived from isolates of Bartonella taylorii (A-1 and A-3), B. grahamii (B-1) and N40 (A-2 and A4) [11]. Two of the new ISR genotypes (A-5 and A-6) could be aligned and were found to be very similar to those obtained for B. taylorii and N40. As these two species possess very similar ISR genotypes, we were unable to specifically assign either of these new genotypes to one or other of these species. However, glt $A$ analysis was able to assign to species the strains from which these two ISR genotypes were derived. A partial glt $A$ sequence that was identical to that of the $B$. taylorii type strain was obtained from three blood samples that had yielded ISR genotype A-5, and a partial glt $A$ sequence identical to that of the N40 representative strain was obtained from three blood samples that had yielded ISR genotype A-6. One of the remaining ISR genotypes (C-2) could be aligned with that of the $B$. doshiae type strain and a partial (654 base pair) glt $A$ sequence comparison revealed $96 \%$ similarity. This value is markedly higher than the similarities of $93 \%$ or lower that are observed between the glt $A$ sequences of different Bartonella species [9].

A previous study found ISRs that could not be meaningfully aligned with one another were derived from different Bartonella species [11]. Thus, on this basis, we assigned the remaining four previously unencountered ISR genotypes to two new putative species. GltA analysis supported this assignment. All three variants of ISR genotype D possessed an identical glt $A$ sequence that shared less than $92 \%$ similarity with those available for other Bartonella species. However, our efforts to obtain a $g l t A$ sequence from the blood sample that yielded ISR genotype E failed and exhaustion of the DNA extract prevented a repeat attempt. In summary, the 12 ISR genotypes were assigned to 5 putatively distinct Bartonella species, as follows: B. taylorii (ISR genotypes A-1, A3, A-5), B. grahamii (B-1), Bartonella N40 (A-2, A-4, A-6), B. doshiae (C-2), Bartonella species D (D-1, D2, D-3) and Bartonella species E (E).

The distribution of these 12 genotypes among the 70 amplification products is presented in Table 1. It should be borne in mind when examining the table that the genotypes were characterized in a random order and without their provenance being known. Thus, the clustering of a single species or strain within a chronological sequence of samples from the same host, for example, was not the result of these isolates themselves being characterized sequentially. The 12 genotypes were not encountered with the same frequency. Species E, for instance, was detected only once, whereas $B$. doshiae (ISR genotype C-2) was amplified from 21 samples and B. grahamii from 20. Bearing this in mind, there is no evidence of any Bartonella species being specific either to one species of host or to one of the two sites. Nor was there any suggestion of any Bartonella species being disproportionately found in either host or at either site. Furthermore, even over the course of the sampling period, more than 1 species of Bartonella was detected from all host individuals, 4 species were detected in the cases of 5 hosts, and from 1 host, 5 were detected. Conversely, with the caveat that the sample size is small, there is a suggestion that at least some strains may be site-specific: B. taylorii ISR genotype A-3 and N40 ISR genotype A-6, for instance, were both isolated on several occasions and from both host species at Rake Hey, but never at Manor Wood.

Chronic infection, over a period of months, was relatively infrequently encountered. In 22 cases, the same species was detected in an animal over 2 or even 3 consecutive months, but more often (42 cases), a species detected during 1 month was no longer there during the next. A species' presence was followed by, in roughly equal proportions, either an infection due to a different species (22 occasions) or by no detectable infection (20 occasions). On no occasion, however 
(with the caveat that mixed infections were occasionally not characterized to strain) was a strain within a species immediately superseded by a different strain of the same species. In 7 of the 12 animals, however, a species detected during the early part of the study but then replaced either by a different species or by no apparent infection, was reencountered in later samples. The delays between initial encounter and re-encounter varied but were as short as 2 months.

Mixed infections were apparently uncommon. Among the four Bartonella species that were common enough for meaningful comparisons to be made ( $B$. taylorii, B. grahamii, N40 and B. doshiae), out of 6 possible comparisons between pairs of species, 5 showed fewer than expected mixed infections, of which 3 came very close to statistical significance (N40 and $B$. doshiae, $\chi_{\underline{1}}^{2}$ (with the Haber correction for continuity $)=3.51, P \leqslant 0.065 ; B$. grahamii and $B$. taylorii, $\chi_{1}^{2}=3.38, P \leqslant 0.07 ; B$. grahamii and $B$. doshiae, $\left.\chi_{1}^{2}=3.26, \quad P \leqslant 0.075\right)$. In line with the patterns of successive infections (above), none of the mixed infections comprised two strains of the same species. In eight instances, a blood sample from an individual was tested in the month preceding a mixed infection. In seven of these, at least one of the species in the mixed infection was also present in the preceding sample. In all 11 mixed infections, a blood sample was tested in the immediately following month. In only five of these were at least one of the species in the mixed infection also present in the subsequent sample. On four occasions both were replaced by a single, different species, whereas on two occasions no subsequent infection could be detected.

The novel partial gltA sequences obtained in this study have been submitted to the GenBank database under the following accession numbers: uncultured $B$. doshiae strain (ISR genotype C-2) (wbs013), AF207827, and uncultured Bartonella spp. D (ISR genotypes D-1, D-2 and D-3) (wbs089), AF207828.

\section{DISCUSSION}

An understanding of the epidemiology of Bartonella species in their natural hosts is important from several perspectives. In terms of public health, the spectrum of bartonella-associated infections continues to expand together with the number of species implicated as pathogens [2]. Very recent work has linked one of the species that exploit UK woodland rodents to human disease [16]. This finding adds weight to the proposition that all Bartonella species are potentially pathogenic to man but that this potential is tempered if contact between man and the species' natural host is seldom made. From an ecological standpoint, the study of bartonella infections in woodland rodents can potentially provide much-needed data to test theoretical models of infectious disease dynamics within naturally susceptible populations and the role of such infections in the regulation of host abundance [1].

More comprehensive follow-up studies will require the testing of large numbers of animals and, due to their simplicity and speed, the methods we have used [11] lend themselves to such work. Nonetheless, even in this preliminary study we have been able to demonstrate, for the first time, a complex in vivo epidemiology for Bartonella species in their natural hosts. Although our findings require confirmation using culture-based methods, they provide clear evidence in support of a very dynamic microbial ecology within the blood of bank voles and wood mice.

The high prevalence of infection identified in horizontal surveys of natural populations of woodland mammals $[7,8]$ combined with studies of experimental infections in rodents has led to the notion that individual animals are susceptible to persistent, long-term bacteraemia rather than high incident rates of infection $[8,17]$. Our findings, however, suggest that this is not typically the case in natural infections. Furthermore, on this basis, the applicability to field epidemiology of data drawn from laboratory-based studies of bartonella infections in other host species may also be limited. Our samples were collected at monthly intervals. Thus, a detected species may have been present in the animal's blood for several weeks. In some animals we observed the same genotype in consecutive samples, which may be interpreted as an extremely chronic infection. However, as the sensitivity of our differentiation method remains unknown, this same genotype may in fact represent different infecting strains. Furthermore, as a positive PCR result is not an indicator of viability, our assay may be prone to overestimation of bacteraemia duration through the detection of remnant DNA from dead or destroyed bartonellae. These uncertainties notwithstanding, however, our results demonstrate that a continuous turnover of specific bacteraemias occurs in most animals, with both chronic and mixed infection being uncommon. 
Our results also indicated that super-infection could only be achieved by a genotype belonging to a different Bartonella species from that of the current infection. This observation is not unexpected if different Bartonella species possess distinct immunogenic antigens during natural infections as they do when grown on axenic media [18]. Thus, immunity to one strain should provide cross-protection against other strains of the same species, but not against strains of other species. However, Kosoy and colleagues [8] reported that naturally infected American rodents developed no detectable antibodies, a finding they subsequently suggested may be due to immunotolerence as a consequence of transplacental infection [19]. It is not known if transplacental transmission occurs in our populations, but the dynamic epidemiology of infections reported here makes it unlikely to be the most common route of infection. It may, in any case, be wrong to accept that axenic immunogen expression by bartonellae accurately reflects that occurring in vivo. In humans, antibodies raised against one infecting species generally cross-react significantly with antigens derived from other (albeit axenically-grown) species [20]. Furthermore, thermal adaptation of bartonellae, as needs to occur in vivo during their transmission from ectoparasite to mammalian host, involves the upregulation of a number of proteins, some of which were identified as being recognized heat-shock proteins with immunogenic properties [21]. Similarly, the ability of bartonellae to adapt to oxidant stress has been demonstrated [22], and this response may also lead to expression of specifically induced immunogens. Despite these concerns, very recent data derived from work on laboratory specific pathogen-free cats has found a lack of heterologous protection between $B$. henselae and $B$. clarridgeiae, and even between different $B$. henselae serogroups/subtypes [23].

Although this study demonstrates there to be a very dynamic epidemiology of Bartonella species in their natural hosts, the effect of these repeated infections on either an individual host or the naturally infected population as a whole remain as yet unknown. Indeed, the lack of humoral response [8] may suggest there to be little or no cost to an individual. Furthermore, despite observing that trypanosome and/or bartonella infections in bank voles were associated with lowered haemocrit levels and enlarged spleens, Wiger [24] concluded that such parasites were of little importance as mortality factors in the population ecology of the rodent. However, recent work on $B$. henselae in cats has suggested that although no immediate and overt manifestations result from infection, the ability of infected cats to become or sustain pregnancy may be compromised [25]. Feore and colleagues [26] have recently shown that cowpox infection in bank voles can also induce pregnancy delay/failure and point out that although having no immediate effect on the individual host, such manifestations have a cost to the natural population as a whole through the expense of lost litters. Drawing from the findings of these two studies, it would be pertinent to determine whether rodent bartonellae have a similar effect on their natural hosts.

\section{REFERENCES}

1. Grenfell BT, Dobson AP. The dynamics of diseases of wildlife. Cambridge: Cambridge University Press, 1993.

2. Birtles RJ, Raoult D. The genera Afipia and Bartonella. In: Schmidt A, ed. Bartonella species as emerging pathogens, with special reference to Bartonella henselae. Basel: Karger AG, 1998: 1-31.

3. Heller R, Riegel P, Hansmann Y, et al. Bartonella tribocorum sp. nov., a new Bartonella species isolated from the blood of wild rats. Int J Syst Bacteriol 1998; 48: 1333-9.

4. Heller R, Kubina M, Mariet P, et al. Bartonella alsatica sp. nov., a new Bartonella species isolated from the blood of wild rabbits. Int J Syst Bacteriol 1999; 49: 283-8.

5. Droz S, Chi B, Steigerwalt AG, Whitney AM, Brenner DJ. Bartonella koehlerae sp. nov., isolated from cats. J Clin Microbiol 1999; 37: 1117-22.

6. Pappalardo BL, Correa MT, York CC, Peat CY, Breitschwerdt EB. Epidemiologic evaluation of the risk factors associated with exposure and seroreactivity to Bartonella vinsonii in dogs. Am J Vet Res 1997; 58: 467-71.

7. Birtles RJ, Harrison TG, Molyneux DH. Grahamella in small woodland rodents in the UK. Isolation, prevalence and host specificity. Ann Trop Med Parasitol 1994; 88: 317-27.

8. Kosoy MY, Regnery RL, Tzianabos T, et al. Distribution, diversity and host specificity of Bartonella in rodents from the south-eastern United States. Am J Trop Med Hyg 1997; 57: 578-88.

9. Birtles RJ, Raoult D. Comparison of partial citrate synthase gene $(g l t A)$ sequences for phylogenetic analysis of Bartonella species. Int J Syst Bacteriol 1996; 46: 891-7.

10. Turner CMR. Seasonal and age distribution of Babesia, Hepatozoon, Trypanosoma and Grahamella species in Clethrionomys glareolus and Apodemus sylvaticus populations. Parasitol 1986; 93: 279-89.

11. Birtles RJ, Hazel SM, Bown K, Raoult D, Begon M, Bennett M. Subtyping of uncultured bartonellae using 
sequence comparison of $16 \mathrm{~S} / 23 \mathrm{~S}$ rRNA intergenic spacer regions amplified directly from infected bloods. Cell Molec Probes 2000; 14: 79-87.

12. Hazel SM, Bennett M, Chantrey J, et al. A longitudunal survey of endemic disease in its wildlife reservoir: cowpox and wild rodents. Epidemiol Infect 2000; 124: 551-62.

13. Hofmeister EK, Kolbert CP, Abdulkarim AS, et al. Cosegregation of a novel Bartonella species with Borrelia burgdorferi and Babesia microti in Peromyscus leucopus. J Infect Dis 1998; 177: 409-16.

14. Roux V, Raoult D. Inter and intra-species identification of Bartonella species. J Clin Microbiol 1995; 33 : 1573-9.

15. Higgins D, Bleasby A, Fuchs R. Clustal V: improved software for multiple sequence analysis. Comput Appl Biosciences 1992; 8: 188-92.

16. Kerkhoff FT, Bergmans AMC, Van Der Zee A, Rothova A. Demonstration of Bartonella grahamii DNA in ocular fluids of a patient with neuroretinitis. J Clin Microbiol 1999; 37: 4034-8.

17. Krampitz HE, Kleinscmidt A. Grahamella Brumpt 1911. Biologische und morphologische untersuchungen. Zeitsch Tropen Parasitol 1960; 11: 336-52.

18. Birtles RJ, Harrison TG, Saunders NA, Molyneux DH. Proposals to unify the genera Grahamella and Bartonella, with descriptions of Bartonella talpae comb. nov., Bartonella peromysci comb. nov., and three new species, Bartonella grahamii sp. nov., Bartonella taylorii sp. nov. and Bartonella doshiae sp. nov. Int J Syst Bacteriol 1995; 45: 1-8.

19. Kosoy MY, Regnery RL, Kosaya OI, Jones DC, Marston EL, Childs JE. Isolation of Bartonella spp. from embryos and neonates of naturally infected rodents. J Wildlife Dis 1998; 34: 305-9.

20. Drancourt M, Mainardi JL, Brouqui P, et al. Bartonella quintana endocarditis in homeless men. New Engl $\mathbf{J}$ Med 1995; 332: 419-23.

21. Haake DA, Summers TA, McCoy AM, Schwartzman W. Heat shock response and groEL sequence of Bartonella henselae and Bartonella quintana. Microbiol 1997; 143: 2807-15.

22. Conley TD, Wack MF, Hamilton KK, Slater LN. Stimulation of angiogenesis and protection from oxidant damage; two potential mechanisms involved in pathogenesis by Bartonella henselae and other Bartonella species. In: Anderson BA, ed. Rickettsial infection and immunity. New York: Plenum Press. 1997: 213-32.

23. Yamamoto K, Chomel BB, Kasten RW, et al. Homologous protection but lack of heterologous-protection by various species and types of Bartonella in specific pathogen-free cats. Vet Immunol Immunopathol 1998; 65: 191-204.

24. Wiger R. Seasonal and annual variations in the prevalence of blood parasites in cyclic species of small rodents in Norway with special reference to Clethrionomys glareolus. Holartic Ecol 1979; 2: 169-75.

25. Guptill L, Slater LN, Wu CC, et al. Evidence of reproductive failure and lack of perinatal transmission of Bartonella henselae in experimentally infected cats. Vet Immunol Immunopathol 1998; 65: 177-89.

26. Feore SM, Bennett M, Chantrey J, Jones T, Baxby D, Begon M. The effect of cowpox virus infection on fecundity in bank voles and wood mice. Proc Royal Soc series B (Biol Sci) 1997; 264: 1457-61. 